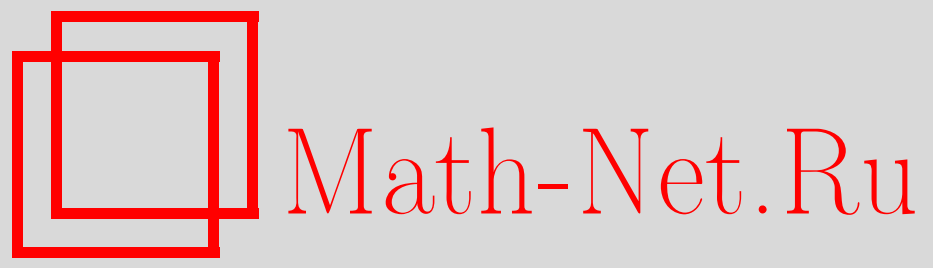

Обцероссийский математический портал

В. П. Маслов, А. Э. Рууге, Пара Лакса для уравнений характеристик осредненных многочастичных операторов, Матем. заметки, 1999, том 66, выпуск 5, 793-796

DOI: https://doi.org/10.4213/mzm1223

Использование Общероссийского математического портала Math-Net.Ru подразумевает, что вы прочитали и согласны с пользовательским соглашением http://www. mathnet.ru/rus/agreement

Параметры загрузки:

IP : 54.89 .56 .158

26 апреля 2023 г., $14: 24: 02$ 


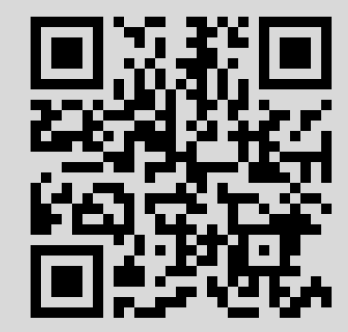




\section{ПАРА ЛАКСА ДЛЯ УРАВНЕНИЙ ХАРАКТЕРИСТИК ОСРЕДНЕННЫХ МНОГОЧАСТИЧНЫХ ОПЕРАТОРОВ}

В.П. Маслов, А. Э. Рууге

Рассмотрим систему уравнений

$$
\begin{aligned}
i \frac{\partial}{\partial t} G\left(x_{1}, x_{2}\right)= & \int d z T\left(x_{2} ; z\right) G\left(x_{1}, z\right)-\int d z T^{*}\left(x_{1} ; z\right) G\left(z, x_{2}\right) \\
& +\int d y d z d w V\left(x_{2}, y ; z, w\right)\left\{-R^{*}\left(x_{1}, y\right) R(w, z)\right. \\
& \left.-G\left(x_{1}, w\right) G^{*}(z, y)+G\left(x_{1}, z\right) G^{*}(w, y)\right\} \\
& -\int d y d z d w V^{*}\left(x_{1}, y ; z, w\right)\left\{-R\left(y, x_{2}\right) R^{*}(z, w)\right. \\
& \left.-G\left(w, x_{2}\right) G^{*}(y, z)+G\left(z, x_{2}\right) G^{*}(y, w)\right\} \\
i \frac{\partial}{\partial t} R\left(x_{1}, x_{2}\right)= & \int d z T\left(x_{2} ; z\right) R\left(x_{1}, z\right)+\int d z T\left(x_{1} ; z\right) R\left(z, x_{2}\right) \\
& +\int d z d w V\left(x_{1}, x_{2} ; z, w\right) R(z, w) \\
& +\int d y d z d w V\left(x_{2}, y ; z, w\right)\left\{-G^{*}\left(x_{1}, y\right) R(w, z)\right. \\
& \left.-G^{*}(z, y) R\left(x_{1}, w\right)+G^{*}(w, y) R\left(x_{1}, z\right)\right\} \\
& +\int d y d z d w V\left(x_{1}, y ; z, w\right)\left\{-G\left(y, x_{2}\right) R(z, w)\right. \\
& \left.-G(y, z) R\left(w, x_{2}\right)+G(y, w) R\left(z, x_{2}\right)\right\}
\end{aligned}
$$

Работа выполнена при финансовой поддержке Российского фонда фундаментальных исследований, грант № 99-01-01198. 
где $G, R$ - неизвестные функции, $x_{1}, x_{2}, y, z, w \in \mathcal{M}, \mathcal{M}$-пространство с мерой, $t \in \mathbb{R} ;$ у функций $G$ и $R$ опущен аргумент $t, T(x ; y), V(x, y ; z, w)$ - заданные обобщенные функции, удовлетворяющие условиям

$$
\begin{gathered}
T(x ; y)=T^{*}(y ; x), \\
V(x, y ; z, w)=V(y, x ; w, z)=V^{*}(z, w ; x, y) .
\end{gathered}
$$

Частный случай уравнений (1), (2) хорошо известен в физической литературе: если наложить на функции $G(x, y)$ и $R(x, y)$ дополнительные условия

$$
G(x, y)=G^{*}(y, x), \quad R(x, y)=-R(y, x),
$$

то уравнения (1), (2) переходят в уравнения, следующие из вариационного принципа Боголюбова для фермионов [1]. Функция $T(x ; y)$ имеет смысл интегрального ядра гамильтониана отдельной частицы $\widehat{T}$, а функция $V(x, y ; z, w)$ - интегрального ядра оператора парного взаимодействия между частицами $\widehat{V}$ :

$$
\widehat{T} \psi(x)=\int d z T(x ; z) \psi(\xi), \quad \widehat{V} \Psi(x, y)=\int d z d w V(x, y ; z, w) \Psi(z, w) .
$$

В работе [2] была дана новая интерпретация уравнений (1), (2) как системы уравнений нестандартных характеристик фермионного осредненного оператора, отвечающего набору операторов

$$
\widehat{\mathcal{H}}_{N}=\sum_{j=1}^{N} \widehat{T}_{x_{j}}+\sum_{1 \leqslant j<k \leqslant N} \widehat{V}_{x_{j} x_{k}}
$$

где $\widehat{\mathcal{H}}_{N}$ действуют в пространстве функций $\psi_{N}\left(x_{1}, \ldots, x_{2}\right) \in L^{2}(\mathcal{M} \times \cdots \times \mathcal{M}), N \in \mathbb{N}$; индексы рядом с операторами $\widehat{T}$ и $\widehat{V}$ обозначают переменные, по которым они применяются. Примерами операторов вида (3) являются оператор Шрёдингера [3] и оператор Лиувилля [4] для системы из $N$ тождественных попарно взаимодействующих частиц. Там же было показано, что в частном случае, когда

$$
\begin{gathered}
T(x ; y)=\left(-\frac{1}{2} \Delta+u(x)\right) \delta(x-y), \\
V(x, y ; z, w)=V(x-y) \delta(x-z) \delta(y-w),
\end{gathered}
$$

где $u$ и $V$ - заданные действительные функции, для уравнений (1), (2) удается найти пару Лакса [5]. Оказывается, что пара Лакса существует и в общем случае. Обозначим

$$
\begin{gathered}
H(x ; y)=T(x ; y)+\int d z d w(V(x, z ; y, w)-V(x, z ; w, y)) G(z, w) \\
B(x ; y)=\int d z d w V(x, y ; z, w) R(z, w) .
\end{gathered}
$$

Tеорема. Коммутатор матрии,

$$
A=\left(\begin{array}{cc}
\widehat{G}-1 / 2 & -\widehat{R}^{*} \\
\widehat{R} & -\widehat{G}^{*}+1 / 2
\end{array}\right), \quad L=\left(\begin{array}{cc}
\widehat{H}^{*} & -\widehat{B}^{*} \\
\widehat{B} & -\widehat{H}
\end{array}\right),
$$

əде $\widehat{G}, \widehat{R}, \widehat{H}$ и $\widehat{B}$ - операторы, задаваемые интегральными ядрами $G, R, H$ и В соответственно, имеет вид

$$
[A, L]=\left(\begin{array}{cc}
\widehat{\alpha} & \widehat{\beta}^{*} \\
\widehat{\beta} & \widehat{\alpha}^{*}
\end{array}\right),
$$


где интегральное ядро $\widehat{\alpha}$ совпадает с правой частью (1), а интегральное ядро $\widehat{\beta}$ совпадает с правой частью (2); звездочка обозначает комплексное сопряжсение.

При исследовании конкретных физических задач достаточно часто встречается ситуация подобная (4), (5), когда функции $T$ и $V$ зависят по части из аргументов как от разностей:

$$
\begin{gathered}
T(x ; y)=T_{0}\left(x^{\prime}-y^{\prime}, \xi ; \eta\right) \\
V(x, y ; z, w)=V_{0}\left(x^{\prime}-y^{\prime}, x^{\prime}-z^{\prime}, y^{\prime}-w^{\prime}, \xi, \eta ; \zeta, \omega\right),
\end{gathered}
$$

где $x=\left(x^{\prime}, \xi\right), y=\left(y^{\prime}, \eta\right), z=\left(z^{\prime}, \zeta\right), w=\left(w^{\prime}, \omega\right), x^{\prime}, y^{\prime}, z^{\prime}, w^{\prime} \in \mathbb{R}^{d}, \xi, \eta, \zeta, \omega \in \mathcal{N}, \mathcal{M}=\mathbb{R}^{d} \times \mathcal{N}$. В этом случае, в уравнениях (1), (2) можно провести подстановку

$$
\begin{aligned}
& G(x, y)=\frac{1}{(2 \pi)^{d}} \int d p G_{p}(\xi, \eta) e^{i p\left(x^{\prime}-y^{\prime}\right)}, \\
& R(x, y)=\frac{1}{(2 \pi)^{d}} \int d p R_{p}(\xi, \eta) e^{i p\left(x^{\prime}-y^{\prime}\right)},
\end{aligned}
$$

где $p \in \mathbb{R}^{d}$, и перейти от исходных уравнений к более простым уравнениям на $G_{p}(\xi, \eta)$ и $R_{p}(\xi, \eta)$.

Обозначим

$$
\begin{aligned}
H_{p}(\xi ; \eta)= & \widetilde{T}(p, \xi ; \eta)+\int d q d \zeta d \omega(\widetilde{V}(0, p,-q, \xi, \zeta ; \eta, \omega) \\
& -\widetilde{V}(q+p,-q, p, \xi, \zeta ; \omega, \eta)) G_{q}(\zeta, \omega), \\
B_{p}(\xi ; \eta)= & \int d q d \zeta d \omega \widetilde{V}(p-q, q,-q, \xi, \eta ; \zeta, \omega) R_{q}(\zeta, \omega)
\end{aligned}
$$

где

$$
\begin{gathered}
\widetilde{T}(p, \xi ; \eta)=\int d y^{\prime} T_{0}\left(y^{\prime}, \xi ; \eta\right) e^{-i p y^{\prime}} \\
\widetilde{V}(p, k, q, \xi, \eta ; \zeta, \omega)=\frac{1}{(2 \pi)^{d}} \int d y^{\prime} d z^{\prime} d w^{\prime} V_{0}\left(y^{\prime}, z^{\prime}, w^{\prime}, \xi, \eta ; \zeta, \omega\right) e^{-i\left(p y^{\prime}+k z^{\prime}+q w^{\prime}\right)}
\end{gathered}
$$

Из приведенной теоремы следует, что для системы уравнений для $G_{p}(\xi, \eta)$ и $R_{p}(\xi, \eta)$ также существует пара Лакса.

СледСтвиЕ. Матрица

$$
A_{p}=\left(\begin{array}{cc}
\widehat{G}_{p}-1 / 2 & -\widehat{R}_{-p}^{*} \\
\widehat{R}_{p} & -\widehat{G}_{-p}^{*}+1 / 2
\end{array}\right)
$$

əде $\widehat{G}_{p}$ и $\widehat{R}_{p}$ - операторь, задаваемые интегральными ядрами $G_{p}(\xi ; \eta)$ и $R_{p}(\xi ; \eta)$ соответственно, удовлетворяет уравнению

$$
i \frac{d}{d t} A_{p}=\left[A_{p}, L_{p}\right]
$$

əde

$$
L_{p}=\left(\begin{array}{cc}
\widehat{H}_{p}^{*} & -\widehat{B}_{-p}^{*} \\
\widehat{B}_{p} & -\widehat{H}_{-p}
\end{array}\right),
$$

$\widehat{B}_{p} и \widehat{H}_{p}$ - операторы, задаваемье интегральными ядрами $B_{p}(\xi ; \eta)$ и $H_{p}(\xi ; \eta)$ соответственно. 


\section{СПИСОК ЦИТИРОВАННОЙ ЛИТЕРАТУРЫ}

1. Боголюбов Н. Н. // Докл. АН СССР. 1958. Т. 119. № 2. С. 244-246. 2. Маслов В. П. // Функцион. анализ и его прилож. 1999 (в печати). 3. Ландау Л. Д., Лифшиц И. М. Квантовая механика. М.: Физматгиз, 1963. 4. Л андау Л. Д., Лифшиц И. М. Механика. М.: Физматгиз, 1958. 5. Манин Ю. И. // Алгебраические аспекты нелинейных дифференциальных уравнений Современные проблемы математики. Т. 11. М.: ВИНИТИ, 1978. С. 5-152.

Московский государственный универсистет им. М.В.Ломоносова 